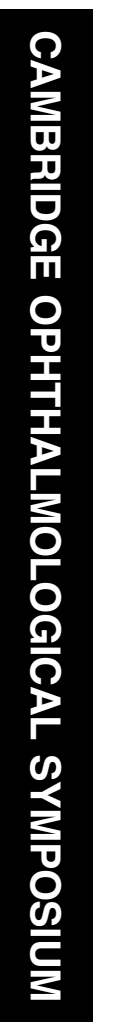

${ }^{1}$ Department of

Ophthalmology and Visual Sciences, University of Illinois Eye and Ear Infirmary, Chicago, IL, USA

${ }^{2}$ Division of Epidemiology and Biostatistics, School of Public Health, University of Illinois at Chicago,

Chicago, IL, USA

Correspondence: EY Tu, Department of Ophthalmology and Visual Sciences, University of Illinois Eye and Ear Infirmary, 1855 West Taylor Street (M/C 648), Room 3.164, Chicago, IL 60612, USA Tel: + 13129968937 ; Fax: + 13123554248 E-mail: etu@uic.edu

Received: 27 September 2011

Accepted in revised form:

12 October 2011

Published online: 16

December 2011

This work was presented at the Cambridge

Ophthalmologic Symposium, September 2011.

\section{Microsporidia and Acanthamoeba: the role of emerging corneal pathogens}

\begin{abstract}
Parasitic organisms are increasingly recognized as human corneal pathogens. A notable increase in both well-defined Acanthamoeba keratitis and a more dramatic increase in reported cases of Microsporidia keratitis have suggested significant outbreaks of parasitic keratitis around the world. Historical and contemporary baselines as well as a familiar associated clinical presentation reinforce the significant outbreak of Acanthamoeba keratitis in the United States. The remarkable rise in cases of Microsporidia keratitis, however, lacks these established baselines and, further, describes a disease that is inconsistent with previous definitions of disease. While a well-defined, abrupt increase strongly suggests temporally related risk factors, most likely environmental, involved in the Acanthamoeba outbreak, the rise in Microsporidia keratitis suggests that increased awareness and improved diagnostic acumen are a significant factor in case ascertainment. Regardless, recent evidence indicates that both parasitic diseases are likely underreported in various forms of infectious keratitis, which may have unrecognized but significant implications in the pathogenesis of both primary protozoal and polymicrobial keratitis. Further understanding of the incidence and interaction of these organisms with current therapeutic regimens and more commonly recognized pathogens should significantly improve diagnosis and alter clinical outcomes.
\end{abstract}

Eye (2012) 26, 222-227; doi:10.1038/eye.2011.315; published online 16 December 2011

Keywords: Acanthamoeba; Microsporidia; parasitic; infectious keratitis; epidemiology

\section{Introduction}

Over the last decade, parasitic organisms have been increasingly associated with various forms
EY Tu ${ }^{1}$ and CE Joslin ${ }^{1,2}$

of external ocular infections worldwide. The term parasite, strictly defined as an organism deriving benefit from a host while providing no benefit in return, could be applied to any corneal pathogen, but is usually reserved for protozoa or other more complex organisms. Several parasitic corneal pathogens have been well described, including Onchocerca, Leishmania, and Trypanosoma, ${ }^{1,2}$ with recent attention focused on notable numbers of Microsporidia keratitis in South Asia and a more modest increase of Acanthamoeba keratitis in the United States as well as other developed countries. $^{3-10}$

Clinically, parasitic infections are usually characterized as chronic and intractable presenting with non-specific findings masquerading as other infectious and non-infectious disease. Despite the recent outbreaks, these infections remain comparatively rare, requiring a substantial clinical suspicion to warrant the specific media and/or special histological expertise for diagnosis. Routine microbiological tests are insensitive. Finally, each of these infections traditionally requires uncommonly available drugs specifically effective against the individual pathogen for resolution. ${ }^{11}$

Altogether, these factors contribute to a delay in the diagnosis that may result in a clinical characterization of the 'classic' presentations used to define cases of parasite-related disease that is biased toward 'later' and poorly treatable disease at the expense of 'earlier' forms of the disease. ${ }^{12}$ An examination of the recent increase in reports of protozoal disease offers an insight into a burden of disease previously unrecognized, but of potentially significant ocular and public health importance both locally and globally.

\section{Recent increases in Microsporidia keratitis}

Microsporidia describes a group of obligate intracellular organisms spanning several 
genera, which reproduce within the host cell by forming spores, which are then released to infect neighboring cells. First described in a Sri Lankan boy in 1973, few further reports appeared in the literature until 1990 when it was first associated with HIV/AIDS infection. ${ }^{13,14}$ Cases were defined as either a vision-limiting, diffuse epitheliitis (Figure 1a) found more commonly in immunocompromised hosts and associated with the genus Encephalitozoon, or a previously described stromal keratitis associated with the genera Vittaforma or Trachipleistophora in immunocompetent hosts. Both were chronic, intractable infections that would occasionally resolve with restoration of immune function and respond variably to topical fumagillin or systemic albendazole. ${ }^{15-17}$ Isolation required incubation with host cells, not easily performed in most laboratories, or direct observation in microbiological smears. With a few exceptions, ${ }^{18}$ Microsporidia keratitis remained primarily a disease of immunocompromised individuals until the first multiple case series of Microsporidia keratitis in immunocompetent individuals were reported from Singapore in 2003 and India in 2005. ${ }^{10,19}$

Subsequently in this geographic region, the number of reported cases increased dramatically with 134 cases reported in 4 years from 2 hospitals in Singapore and even greater numbers reported from various centers in India, which, at face value, suggested an outbreak of the disease. ${ }^{8,9}$ The CDC outlines, however, 10 steps that are
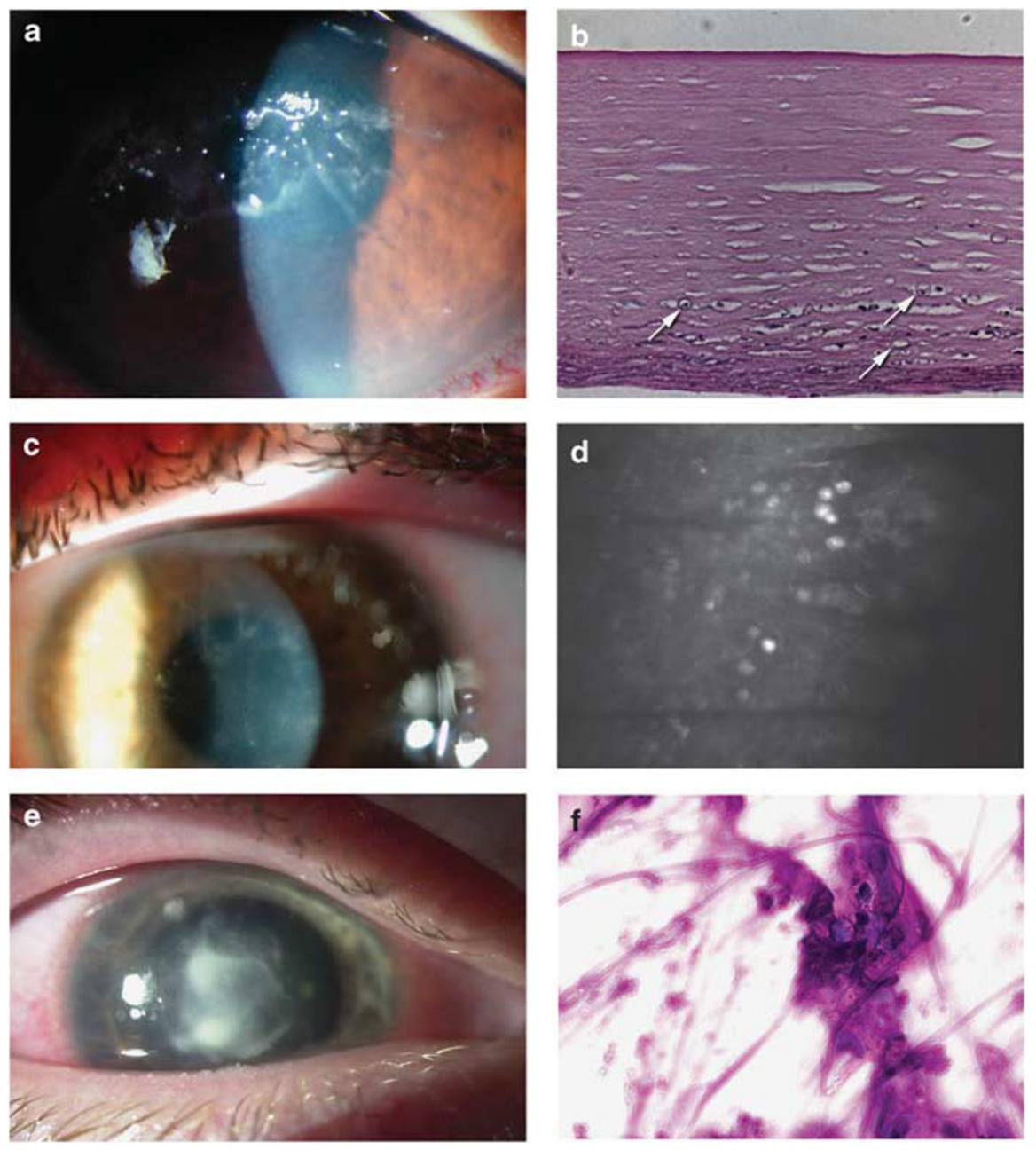

Figure 1 (a) A slit lamp photo of superficial microsporidial keratitis in an AIDS patient. (b) Photo of a histopathological specimen from a therapeutic penetrating keratoplasty performed for Acanthamoeba keratitis showing both full and empty cysts (arrows) in the posterior third of the stroma. (c) Slit lamp photo of epithelial Acanthamoeba keratitis with radial keratoneuritis. (d) Confocal microscopy of the same patient (c) demonstrating round double-walled cysts with a bright center. (e) A case of chronic stromal keratitis associated with Acanthamoeba. (f) Diff-Quick smear of a corneal scraping of the same patient (e) with a single characteristic double-walled cyst centrally with multiple pores. 
integral to an outbreak investigation. ${ }^{20}$ The initial steps involve confirming the existence of an outbreak, specifically, creating a case definition, confirming that the cases are 'real' and, thereby, establishing a background rate of disease. Although the vast majority of these recent cases reported are supported by histological confirmation, several features are inconsistent with our prior understanding of the presentation and course of Microsporidia keratitis. Specifically, these cases are almost exclusively found in immunocompetent individuals and, although previous cases were chronic, difficult to manage, and responded variably to only specific drugs, recent cases have resolved with a wide array of commonly available topical ophthalmic antibiotics $^{8}$ or with no therapy in a period of a few weeks. ${ }^{21}$

While the number of cases of Microsporidia reported has been remarkable, this new case definition suggests that a significant contribution of recognition bias may potentially exist. For example, its non-specific presentation and self-limited nature was routinely mistaken for seasonal outbreaks of presumed viral epidemic keratoconjunctivitis in India. ${ }^{22}$ The shorter course, the self-limited nature of many, if not most, of these infections, and their response to non-specific empiric therapy would all be expected to further mask the number of true number of cases detected. This not only suggests that the more recent disease attributed to Microsporidia is currently underreported, but also likely to be previously more severely underreported. The strikingly different case definition makes it difficult to establish the existence or scope of a Microsporidia keratitis outbreak because of the lack of congruous, contemporary or historical controls utilizing the same case definition.

Clearly, the involvement of Microsporidia in corneal disease is more common now than previously understood, but to what degree this represents an outbreak or increased diagnostic acumen remains unclear. An accurate characterization of the disease burden and morbidity of Microsporidia keratitis and an understanding of its environmental source are important to quantify its unrecognized role in ocular disease and its future prevention. Efforts to control the disease may be most dependent on its perceived morbidity if the underlying source is constant and not easily modifiable.

\section{Recent increases in Acanthamoeba keratitis}

Similar to Microsporidia keratitis, Acanthamoeba keratitis was first described in 1973 with few additional cases reported until a strong association with soft contact-lens wear was recognized in the early $1980 \mathrm{~s} .{ }^{23-25}$ Its subsequent history has, however, been characterized by a series of outbreaks in developed countries where contact-lens wear, still its most strongly associated risk factor, is common. ${ }^{26-29}$ In the United States, analysis of an Acanthamoeba keratitis outbreak in the late 1980s calculated an acute incidence of approximately two cases per million contact lens wearers per year. ${ }^{30}$ Similarly, comprehensive studies in the UK point to a much higher incidence of 17.53 per million rigid lens wearers and 21.14 per million soft lens wearers per year. ${ }^{31}$ While no consistent background surveillance has been performed, a high level of detection at tertiary care facilities where the diagnosis could be made, appropriate treatment initiated and cases aggregated has been assumed because of its chronic nature and almost universal resistance to commonly available ophthalmic anti-infectives.

An increase in the number of cases of Acanthamoeba starting in 2003 in Chicago and 2004 elsewhere in the US has been noted in several recent studies. ${ }^{5,32}$ Similar questions have been raised as to whether this increase constitutes an outbreak or a previously unrecognized background rate. Applying the same CDC criteria, the clinical aspect of the case definition for Acanthamoeba keratitis, although varied, has remained relatively consistent among past and present studies ranging from predominantly epithelial disease to deep stromal keratitis (Figures $1 \mathrm{~b}-\mathrm{f}$ ) sometimes associated with extraocular manifestations and occurring predominantly in contact lens wearers. Gross calculations of incidence in the Chicago metropolitan area approach 20 cases per million contact lens users per year, an order of magnitude increase over the historical outbreak incidence of the late 1980s, but very similar to that seen in the UK.

The question as to whether these cases are 'real' is more controversial as the method of diagnosis in most prior US studies has relied heavily on confocal imaging (Figure 1d) with low rates of microbiological support (Figure 1f). ${ }^{28,33}$ Our own experience with confocal microscopy has been very supportive of its use, however, it should be noted that it was not used as a the sole criteria for diagnosis and that culture rates were at least equal to if not exceeding those published in other international studies. ${ }^{34}$ Confocal microscopy remains a useful tool for rapid diagnosis, but correlation varies significantly and should be individually validated..$^{35}$ Applying the stricter criteria of microbiological evidence alone, the increase in cases in Chicago would still represent a significant increase over previously understood rates in the United States. Further, the initial CDC surveys captured only culture-positive cases over the last decade indicating that the rise in these centers was real, applying this narrow diagnostic criterion within this time frame. ${ }^{3,36}$ Taken together, a consistent case definition, confirmation of real cases, and various, 
but consistent contemporary and historical background rates, it appears that the recent rise in cases of Acanthamoeba keratitis in the United States meets the criteria of a true outbreak.

Once established as an outbreak, two case-control investigations identified a significantly elevated risk from the use of AMO Complete MoisturePlus compared with other disinfection systems as well as some hygienerelated factors, including solution reuse (topping off), showering in lenses, and so on. ${ }^{3,37}$ The abrupt rise in cases, however, makes it unlikely that a radical change in hygiene habits was solely responsible and, further, the large number of cases not using AMO Complete MoisturePlus still constituted a sizable increase over the known background rate of infection making it also unlikely the sole cause. ${ }^{37}$ Focus on other contemporaneously introduced risk factors including the labeling change for the use of multipurpose solutions allowing the omission of lens rubbing as well as the introduction and subsequent market dominance of silicone hydrogel lenses in the contact lens market have similarly not yielded an explanation. ${ }^{3,11}$ The lack of a specific lens-related risk factor and the significant increase across all solution systems and lens types suggests an increase in exposure to Acanthamoebae characteristic of water contamination seen with almost all previous outbreaks. Our ongoing studies in Chicago continue to demonstrate a geographic-based pattern of disease consistent with the domestic water distribution system as well as a higher rate of Acanthamoeba isolation from domestic taps than previously described. ${ }^{38}$ As expected, although surveillance subsequent to the recall of AMO Complete MoisturePlus in 2007 demonstrated a modest decrease in the number of cases initially, the lack of reduction of Acanthamoeba keratitis cases to baseline levels has prompted another CDC case-control study that was initiated and completed earlier in 2011.

\section{The potential role of unrecognized protozoal keratitis}

As noted previously, the assumption has been, in the case of Acanthamoeba keratitis, that case ascertainment is high or nearly complete because all cases would eventually reach a site where case aggregation could occur. ${ }^{11}$ Recent evidence suggests, however, that Acanthamoeba isolated in the presence of a bacterial keratitis may not require specific therapy for resolution. We found this to be the case in two of our own patients with pseudomonas keratitis, which resolved with topical antibacterial therapy alone before Acanthamoeba cultures were reported positive. This phenomenon has also been reported elsewhere on a larger scale. ${ }^{39}$ Similar to the altered case definition of recent cases of Microsporidia keratitis, these cases of Acanthamoeba keratitis are likely to be undetected as they did not require specific therapy and had none of the clinical characteristics 'classically' associated with Acanthamoeba keratitis.

For Acanthamoeba and Microsporidia, it would seem that the clinical significance of a self-limited or easily treatable infestation would be questionable. This may, however, be important for several reasons. If these organisms are nonpathogenic, then microbiological and especially indirect methods of diagnosing Acanthamoeba or Microsporidia keratitis, including molecular methods, would be difficult to validate. It is known that Acanthamoebal cysts survive poorly in axenic environments. ${ }^{40}$ If antibacterial treatment can eliminate certain strains of Acanthamoebae either through elimination of its bacterial food source or, alternatively, through direct antiAcanthamoebal activity, it would be important to test the anti-Acanthamoebal activity of ophthalmic antibiotics to better tailor empiric therapy of presumed infectious keratitis.

More importantly, perhaps, recent molecular studies have demonstrated that normal mucosal surfaces, including the eye, have a diverse biome undetectable through traditional microbiological methods. ${ }^{41,42}$ The more common presence of undetected organisms, like Acanthamoebae, in routine infectious keratitis could significantly impact our understanding of its pathogenesis. Polymicrobial keratitis, in general, and in combination with Acanthamoebae specifically is known to pursue a more aggressive clinical course. ${ }^{43}$ Acanthamoebae are well documented to be a co-pathogen with viral, bacterial, fungal, and other protozoal organisms as well as a host for a number of pathogenic bacterial endosymbionts, including pseudomonas and legionella. ${ }^{44}$ The impact of these interactions on the often variable clinical course of Acanthamoeba keratitis and even routine bacterial keratitis may produce new therapeutic strategies to improve outcomes of infectious keratitis.

In summary, both Microsporidia and Acanthamoeba keratitis have a larger and increasingly recognized role in infections of the cornea. Recent evidence suggests that their true incidence may be significantly underreported, which not only underestimates their public health burden but also masks the scope of their associated morbidity. A better understanding of the modifiable risk factors involved in the individual outbreaks and of their role in corneal disease is needed.

\section{Conflict of interest}

The authors declare no conflict of interest. 


\section{References}

1 Pearlman E, Gillette-Ferguson I. Onchocerca volvulus, Wolbachia and river blindness. Chem Immunol Allergy 2007; 92: 254-265.

2 Lam S. Keratitis caused by leishmaniasis and trypanosomiasis. Ophthalmol Clin N Am 1994; 7(4): 635-639.

3 Verani JR, Lorick SA, Yoder JS, Beach MJ, Braden CR, Roberts JM et al. National outbreak of Acanthamoeba keratitis associated with use of a contact lens solution, United States. Emerg Infect Dis 2009; 15(8): 1236-1242.

4 Tu EY, Joslin CE. Recent outbreaks of atypical contact lensrelated keratitis: what have we learned? Am J Ophthalmol 2010; 150(5): 602-608.e2.

5 Thebpatiphat N, Hammersmith KM, Rocha FN, Rapuano CJ, Ayres BD, Laibson PR et al. Acanthamoeba keratitis: a parasite on the rise. Cornea 2007; 26(6): 701-706.

6 Joslin CE, Tu EY, Shoff ME, Booton GC, Fuerst PA, McMahon TT et al. The association of contact lens solution use and Acanthamoeba keratitis. Am J Ophthalmol 2007; 144(2): 169-180.

7 Joslin CE, Tu EY, McMahon TT, Passaro DJ, Stayner LT, Sugar J. Epidemiological characteristics of a Chicago-area Acanthamoeba keratitis outbreak. Am J Ophthalmol 2006; 142(2): 212-217.

8 Loh RS, Chan CM, Ti SE, Lim L, Chan KS, Tan DT. Emerging prevalence of microsporidial keratitis in Singapore: epidemiology, clinical features, and management. Ophthalmology 2009; 116(12): 2348-2353.

9 Joseph J, Sridhar MS, Murthy S, Sharma S. Clinical and microbiological profile of microsporidial keratoconjunctivitis in southern India. Ophthalmology 2006; 113(4): 531-537.

10 Chan CM, Theng JT, Li L, Tan DT. Microsporidial keratoconjunctivitis in healthy individuals: a case series. Ophthalmology 2003; 110(7): 1420-1425.

11 Tu EY, Joslin CE. Recent outbreaks of atypical contact lens-related keratitis: what have we learned? Am J Ophthalmol 2010; 150(5): 602.e2-608.e2.

12 Tu EY. Acanthamoeba and other parasitic corneal infections. In: Krachmer JH, Mannis M and Holland EJ (eds). Cornea, 3rd ed. Mosby Elsevier, 2011, pp 1023-1032.

13 Friedberg DN, Stenson SM, Orenstein JM, Tierno PM, Charles NC. Microsporidial keratoconjunctivitis in acquired immunodeficiency syndrome. Arch Ophthalmol 1990; 108(4): 504-508.

14 Ashton N, Wirasinha PA. Encephalitozoonosis (nosematosis) of the cornea. Br J Ophthalmol 1973; 57: 669-674.

15 Diesenhouse MC, Wilson LA, Corrent GF, Visvesvara GS, Grossniklaus HE, Bryan RT. Treatment of microsporidial keratoconjunctivitis with topical fumagillin. Am J Ophthalmol 1993; 115(3): 293-298.

16 Didier ES, Rogers LB, Brush AD, Wong S, Traina-Dorge V, Bertucci D. Diagnosis of disseminated microsporidian Encephalitozoon hellem infection by PCR-Southern analysis and successful treatment with albendazole and fumagillin. J Clin Microbiol 1996; 34(4): 947-952.

17 Martins SA, Muccioli C, Belfort Jr R, Castelo A. Resolution of microsporidial keratoconjunctivitis in an AIDS patient treated with highly active antiretroviral therapy. Am J Ophthalmol 2001; 131(3): 378-379.

18 Silverstein BE, Cunningham Jr ET, Margolis TP, Cevallos V, Wong IG. Microsporidial keratoconjunctivitis in a patient without human immunodeficiency virus infection. Am J Ophthalmol 1997; 124(3): 395-396.

19 Vemuganti GK, Garg P, Sharma S, Joseph J, Gopinathan U, Singh S. Is microsporidial keratitis an emerging cause of stromal keratitis? A case series study. BMC Ophthalmol 2005; 5: 19.

20 Reingold AL. Outbreak investigations - a perspective. Emerg Infect Dis [serial on the Internet]. [Web page] 1998 [cited 2011 09.22]. Available from:http://wwwnc.cdc.gov/ eid/article/4/1/98-0104.htm.

21 Sanjay S. Clinical trial of $0.02 \%$ polyhexamethylene biguanide $v s$ placebo in the treatment of microsporidial keratoconjunctivitis. Am J Ophthalmol 2011; 151(1): 183; author reply.

22 Das S, Sharma S, Sahu SK, Nayak SS, Kar S. New microbial spectrum of epidemic keratoconjunctivitis: clinical and laboratory aspects of an outbreak. Br J Ophthalmol 2008; 92(6): 861-862.

23 Naginton J, Watson PG, Playfair TJ, McGill J, Jones BR, Steele AD. Amoebic infection of the eye. Lancet 1974; 2(7896): 1537-1540.

24 Jones BR, McGill JI, Steele AD. Recurrent suppurative kerato-uveitis with loss of eye due to infection by Acanthamoeba castellani. Trans Ophthalmol Soc UK 1975; 95(2): 210-213.

25 Moore MB, McCulley JP, Luckenbach M, Gelender H, Newton C, McDonald MB et al. Acanthamoeba keratitis associated with soft contact lenses. Am J Ophthalmol 1985; 100(3): 396-403.

26 Stehr-Green JK, Bailey TM, Brandt FH, Carr JH, Bond WW, Visvesvara GS. Acanthamoeba keratitis in soft contact lens wearers. A case-control study. JAMA 1987; 258(1): 57-60.

27 Bacon AS, Frazer DG, Dart JK, Matheson M, Ficker LA, Wright P. A review of 72 consecutive cases of Acanthamoeba keratitis, 1984-1992. Eye (Lond) 1993; 7(Part 6): 719-725.

28 Mathers WD, Sutphin JE, Folberg R, Meier PA, Wenzel RP, Elgin RG. Outbreak of keratitis presumed to be caused by Acanthamoeba. Am J Ophthalmol 1996; 121(2): 129-142.

29 Radford CF, Lehmann OJ, Dart JK. Acanthamoeba keratitis: multicentre survey in England 1992-1996. National Acanthamoeba Keratitis Study Group. Br J Ophthalmol 1998; 82(12): 1387-1392.

30 Schaumberg DA, Snow KK, Dana MR. The epidemic of Acanthamoeba keratitis: where do we stand? Cornea 1998; 17(1): 3-10.

31 Radford CF, Minassian DC, Dart JK. Acanthamoeba keratitis in England and Wales: incidence, outcome, and risk factors. Br J Ophthalmol 2002; 86(5): 536-542.

32 Joslin CE, Tu EY, McMahon TT, Passaro DJ, Stayner LT, Sugar J. Epidemiological characteristics of a Chicago-area Acanthamoeba keratitis outbreak. Am J Ophthalmol 2006; 142(2): 212-217.

33 Parmar DN, Awwad ST, Petroll WM, Bowman RW, McCulley JP, Cavanagh HD. Tandem scanning confocal corneal microscopy in the diagnosis of suspected Acanthamoeba keratitis. Ophthalmology 2006; 113(4): 538-547.

34 Tu EY, Joslin CE, Sugar J, Booton GC, Shoff ME, Fuerst PA. The relative value of confocal microscopy and superficial corneal scrapings in the diagnosis of Acanthamoeba keratitis. Cornea 2008; 27(7): 764-772.

35 Hau SC, Dart JK, Vesaluoma M, Parmar DN, Claerhout I, Bibi K et al. Diagnostic accuracy of microbial keratitis with 
in vivo scanning laser confocal microscopy. $\mathrm{Br} J$ Ophthalmol 2010; 94(8): 982-987.

36 Centers for Disease Control and Prevention (CDC). Acanthamoeba keratitis multiple states, 2005-2007. MMWR Morb Mortal Wkly Rep 2007; 56(21): 532-534.

37 Joslin CE, Tu EY, Shoff ME, Booton GC, Fuerst PA, McMahon TT et al. The association of contact lens solution use and Acanthamoeba keratitis. Am J Ophthalmol 2007; 144(2): 169-180.

38 Joslin CE, Tu EY, Shoff ME, Anderson RJ, Davis FG. Shifting distribution of Chicago-area Acanthamoeba keratitis cases. Arch Ophthalmol 2010; 128(1): 137-139.

39 Cervantes LJ, Shih CY, Ritterband DC, Shah M, Seedor JA, Udell IJ. Resolution of Acanthamoeba Keratoconjunctivitis Without the Use of Anti-Acanthamoebal Therapy. Ocular Microbiology and Immunology Group: San Francisco, CA, 2009. http://eyemicrobiology.upmc.com/2009 Abstracts/2009 OMIG Abstract 9.html.
40 Penland RL, Wilhelmus KR. Comparison of axenic and monoxenic media for isolation of Acanthamoeba. J Clin Microbiol 1997; 35(4): 915-922.

41 Muthappan V, Lee AY, Lamprecht TL, Akileswaran L, Dintzis SM, Lee C et al. Biome representational in silico karyotyping. Genome Res 2011; 21(4): 626-633.

42 Dong Q, Brulc JM, Iovieno A, Bates B, Garoutte A, Miller D et al. Diversity of bacteria at healthy human conjunctiva. Invest Ophthalmol Vis Sci 2011; 52(8): 5408-5413.

43 Tu EY, Joslin CE, Nijm LM, Feder RS, Jain S, Shoff ME. Polymicrobial keratitis: Acanthamoeba and infectious crystalline keratopathy. Am J Ophthalmol 2009; 148(1): 13-19.e2.

44 Iovieno A, Ledee DR, Miller D, Alfonso EC. Detection of bacterial endosymbionts in clinical Acanthamoeba isolates. Ophthalmology 2010; 117(3): 445-452, 52 e1-e3. 